\title{
Quantification of efavirenz, emtricitabine and tenofovir in plasma from HIV- positive pregnant women. Analytical development and clinical application
}

\author{
Alicia Ramirez Ramirez ${ }^{1}$, Anahi S. Singuenza Ortega ${ }^{2}$, Zayra Garcia Flores ${ }^{3}$, Jessica Hernandez \\ Pineda ${ }^{1}$, Noemi Plazola Camacho ${ }^{1}$, Ricardo Figueroa Damian ${ }^{1}$ \\ ${ }^{1}$ Infectologia eInmunologia Perinatal, Instituto Nacional de Perinatologia, Mexico, ${ }^{2}$ Facultad de Quimica, Universidad \\ Nacional Autonoma de Mexico, Mexico, ${ }^{3}$ Facultad de Estudios Superiores Cuatitlan, Universidad Nacional Autonama \\ de Mexico, Mexico
}

The most relevant strategy to avoid mother-to-child transmission is the highly active antiretroviral therapy, HAART, during pregnancy. Nowadays, has been reported the use of combinations of ARV as efavirenz, EFV, emtricitabine, FTC, and tenofovir, TDF; administered in a fixed dose combination called ATRIPLA. Documented reports supported EFV teratogenicity effects in the preclinical phase, so the Food and Drug Administration, FDA,classified it as a category D drug. Also, physiological changes in these patients can compromise the therapeutic safety and efficacy of HAART. During 2017, in the InstitutoNacional de Perinatologia, almost 30 percentof HIV-positive pregnant women arrived in the last trimester of pregnancy, using ATRIPLA as HAART. Our primary goal is to develop a chromatographic method with acceptable sensibility, efficiency and reliability, all of them validated by Mexican standards, NOM-177-SSA1-2013 and FDA guidelines, to quantify the ARV drugs from ATRIPLA in pregnant patients and correlated with any adverse outcome in the neonates.

Methods.Transitions of each ARV drug were detected by multiple-reaction-monitoring and positive electrospray for EFV 316.15-244.03, for FTC 248.15-130.00, and TDF 288.20-176.08. To separate the ARV drugs we used an AcquityCSH C18 column, $2.1 \mathrm{umx} 50 \mathrm{~mm}$, and $1.7 \mathrm{um}$, using methanol-ammonium formate, ten microMolar gradient, in two minutes and injections of $3 \mathrm{uL}$. We quantify six plasma samples from pregnant patients receiving ATRIPLA. Extraction of ARV drugs from biological matrix was through protein precipitation with formic acid $0.2 \% \mathrm{MeOH}$. This development was validated under Mexican standards and in consensus with the FDA.

Results. The method developed was validated for a concentration range in ng-mL, 23-3450, 24-3600, and 3-450 for EFV, FTC, and TDF respectively. The matrix effect was less to 15\%. The plasma samples from patients presented concentrations of ARV drugs within the quantisation ranges with a high inter-individual variability.

Conclusions. This UPLC MS-MS methodology developed and validated to use proved to be useful to simultaneous quantify EFV, FTC and TDF with acceptable sensitivity and accuracy, according to the national and international guidelines. This assay could be found application in the improvement of the therapeutic monitoring of ATRIPLA, supporting the creation of pharmacological surveillance programmes for VIH-positive pregnant women receiving any HAART in our Institute. 